E3S Web of Conferences 1, 22002 (2013)

DOI: $10.1051 / \mathrm{e} 3$ sconf/20130122002

(C) Owned by the authors, published by EDP Sciences, 2013

\title{
Monetary Valuation of Health Risks of Heavy Metals: State-of-the-art and some gaps
}

\author{
M. Ščasný ${ }^{1}$ and V. Máca ${ }^{2}$ \\ ${ }^{1}$ Charles University Environment Center, Charles University Prague, José Martího 2/407, 16200 Praha 6, CZECH \\ REPUBLIC, milan.scasny@czp.cuni.cz \\ ${ }^{2}$ Charles University Environment Center, Charles University Prague, José Martího 2/407, 16200 Praha 6, CZECH \\ REPUBLIC, vojtech.maca@czp.cuni.cz
}

\begin{abstract}
Health Impact Assessment (HIA) is a fundamental part of modern decision-making process, in particular policies that affect releases, exposures or doses of pollutants including heavy metals. Traditionally, HIA focus is on risk assessment and securing safe levels of pollution exposure or dose. Only recently HIA has extended its focus to include evaluation of health effects in monetary terms that allows for a comprehensive Cost-Benefit Analysis, a widely used quantitative decision support tool to assess desirability of policies and projects based on economic rationale. The aim of this contribution is to review the state-of-the-art in valuation of health impacts related to heavy metals and discuss some gaps faced here. We start by introducing concept of risk in valuation literature, related mortality denominator, Value of Statistical Life (VSL) and difficulties in deriving values for chronic health conditions. We then overview valuation of various health outcomes in five broad impact categories - carcinogenicity, dose toxicity, sensitization, effects on fertility and developmental toxicity and discuss difficulties in translating risk assessment and toxicological research findings of human health effects of heavy metal exposure/dose to changes in welfare in such a way that is comprehensible for general population. We briefly examine and discuss numerous issues and gaps in establishing such links between exposure and welfare impacts in the context of a study commissioned by European Chemicals Agency. The study's goal is to deliver EU-wide values of willingness to pay for avoiding adverse health outcomes due to exposure to chemicals. We emphasize the necessity of such discussion among experts from different areas for the results to be useful for decision-making related to regulation of (not only) heavy metals releases to the environment.
\end{abstract}

Key words: Health Impact Assessment, Cost-Benefit Analysis, health benefit valuation, health endpoints, Value of Statistical Life, willingness-to-pay.

This is an Open Access article distributed under the terms of the Creative Commons Attribution License 2.0, which permits unrestricted use, distribution, and reproduction in any medium, provided the original work is properly cited. 